\title{
Characteristics of Plasma Dynamics in Current Sheets Formed in Helium Plasma
}

\author{
Anna G. Frank ${ }^{1, *}$, Natalya P. Kyrie ${ }^{1}$, Sergey N. Satunin ${ }^{1}$ and Sergey A. Savinov ${ }^{1,2}$ \\ 1 Prokhorov General Physics Institute of the Russian Academy of Sciences, 119991 Moscow, Russia; \\ natalya.kyrie@yandex.ru (N.P.K.); satosn@yandex.ru (S.N.S.); s.a.savinov@mail.ru (S.A.S.) \\ 2 P.N. Lebedev Physical Institute of the Russian Academy of Sciences, 119991 Moscow, Russia \\ * Correspondence: annfrank@fpl.gpi.ru
}

Citation: Frank, A.G.; Kyrie, N.P.;

Satunin, S.N.; Savinov, S.A.

Characteristics of Plasma Dynamics in Current Sheets Formed in Helium Plasma. Universe 2021, 7, 400. https://doi.org/10.3390/ universe7110400

Academic Editors: Olga V. Khabarova and Gregory D. Fleishman

Received: 21 September 2021

Accepted: 20 October 2021

Published: 23 October 2021

Publisher's Note: MDPI stays neutral with regard to jurisdictional claims in published maps and institutional affiliations.

\begin{abstract}
The characteristic features of plasma acceleration in the current sheets are discussed on the basis of an analysis of the structure of electrodynamic forces at successive stages of the evolution of the current sheets formed in the plasma with helium ions. Of particular interest is the generation of reverse currents at the side edges of the sheet and the appearance of forces, which are braking previously accelerated plasma flows.
\end{abstract}

Keywords: current sheet; laboratory experiment; plasma acceleration; reverse currents; slow down of plasma flows

\section{Introduction}

The formation of current sheets with high concentration of magnetic energy in spatially separated regions of magnetized plasma and the possibility of a rapid release of energy via magnetic reconnection have been intensively studied over the past decades. According to modern concepts, the dynamics of current sheets provide a basis for various flare-type phenomena including Solar flares and flares on other stars, substorms in the magnetospheres of the Earth and other planets, and disruption instability in tokamak plasma [1-5].

Along with the theoretical research, the dynamics of current sheets and magnetic reconnection are studied in dedicated laboratory experiments. These experiments, among other factors, can provide laboratory modeling of non-stationary astrophysical phenomena [6-12]. Laboratory experiments are carried under highly controlled and reproducible conditions and use modern methods of plasma diagnostics, which allows for the relation of plasma dynamics to the evolution of magnetic fields, currents, and electrodynamic forces in current sheets [11-16].

The initial conditions for the formation of current sheets in laboratory experiments can be established within a relatively wide range, therefore providing the current sheets of different structures, much like the current sheets in natural conditions (for example, in the Earth's magnetosphere). In particular, by changing the mass of ions in plasma, we can bring about changes in both the relative thickness of the sheet and the role of the Hall effect in plasma dynamics [14,15]. In plasma with heavy ions, we obtain "thin" sub-ionic current sheets with a thickness of the order of the ion inertial length. In the lighter-ions plasma, "thick" current sheets are usually formed with a thickness exceeding several times the ion inertial length $[14,15,17]$.

Magnetic energy accumulated in the vicinity of the metastable current sheet can be transformed into thermal energy and into the energy of high-speed flows of plasma [18-20]. Plasma is accelerated along the surface of the current sheet mainly under the action of the Ampère forces that are initially directed from the central region of the sheet to its edges on both sides $[11,21]$. In some cases, plasma acceleration can be spatially inhomogeneous in 
the normal direction to the sheet, and higher acceleration can be at some distance from the middle plane of the sheet, in regions of low plasma density [11,22,23].

Accelerated plasma flows along the current sheet surface can result in exciting the reverse currents at the sheet edges. This effect can significantly change the dynamic processes in the current sheets, including the process of plasma acceleration [24].

This paper is focused on the experimental research of plasma dynamics in current sheets produced in helium plasma in two-dimensional magnetic fields with a null line of the X-type. The basic parameters of plasma and current sheets were obtained by spectroscopic methods and magnetic measurements. The energy of accelerated plasma flows is correlated with the structure of electrodynamic forces at successive stages of the current sheet evolution. Of particular interest is the first registration of the reverse currents in current sheets formed in the helium plasma. Excitation of reverse currents gives rise to additional Ampère forces that slow down previously accelerated plasma flows.

\section{Experimental Setup and Methods of Diagnostics}

Plasma dynamics in the current sheets were studied with the experimental setup CS-3D (Figure 1) [7,25,26]. A quartz vacuum chamber (18 cm in diameter, $100 \mathrm{~cm}$ long) was filled with helium gas up to pressure of $320 \mathrm{mTorr}$. A system of straight current-carrying conductors placed outside the vacuum chamber excited a quasistationary 2D magnetic field with the null line of the X-type at the $z$ axis:

$$
\vec{B}=\left\{B_{X}^{0} ; B_{Y}^{0} ; B_{Z}^{0}\right\}=\{h y ; h x ; 0\} .
$$

The null line was aligned with the chamber axis. The magnetic field gradient $h$ in the $x y$-plane was established equal to $0.5 \mathrm{kG} / \mathrm{cm}$. The initial plasma in the magnetic field (1) was produced using the $\Theta$-discharge with strong preliminary ionization, and the initial plasma was almost homogeneous in the $z$ direction. Then, the plasma current $J_{Z}$ was excited by applying the impulsive voltage $U_{Z}$ between two grid electrodes that were arranged at the chamber ends on the distance of $\Delta z=60 \mathrm{~cm}$ from each other. The time dependence of the plasma current $J_{Z}(t)$ was close to sinusoid, with the amplitude $\approx 45 \mathrm{kA}$ and the half-period $T / 2=6.3 \mu \mathrm{s}$.

The current $J_{Z}$ initiated 2 D plasma flows in the magnetic field $\vec{B}$, and gave rise to the current sheet formation during the first 1-1.5 $\mu \mathrm{s}$. The typical transverse sizes of the current sheet were: $2 \delta x \approx 12-17 \mathrm{~cm}$ (width) and $2 \delta y \approx 1.5-3.5 \mathrm{~cm}$ (thickness), at the level of $0.1 \cdot j_{z}^{0}$; here, $j_{z}^{0}$ is the current density at the middle plane of the sheet $[27,28]$. The sheet length along the direction of the current was equal to $\Delta z$.

Plasma flows in the $x y$-plane resulted in increasing the electron density in the middle plane of the current sheet. This density exceeded several times the densities of both the initial plasma and the surrounding plasma $[29,30]$.

The velocities of the helium ions and the electron densities were obtained by registration of the profiles of two spectral lines: the He II $468.6 \mathrm{~nm}$ (the $n=3 \rightarrow n=2$ transition, $P_{\alpha}$ ) and the He II $320.3 \mathrm{~nm}$ (the $n=4 \rightarrow n=2$ transition, $P_{\beta}$ ). Analyzing the spectral data, we took into account that the broadening of the He II $P_{\alpha}$ spectral line was caused by both the Doppler and Stark effects, while the broadening of the He II $P_{\beta}$ line was due mainly to the Stark effect [31,32]. The difference between the constants of the Doppler and Stark broadenings of these spectral lines allowed for calculation of the plasma parameters in the sheet; for details see [20,33].

The optical part of the setup included two channels for simultaneous registration of plasma emission in two mutually perpendicular directions. In the " $z$-channel," the radiation was collected from the central quasi-cylindrical region D1 $(\varnothing \sim 1.5 \mathrm{~cm})$, extended along the direction of the plasma current, shown in Figure 1. This channel was used to determine the plasma density and the ions' thermal velocities in the central region of the current sheet, since the directed plasma motions occur predominantly in the $x y$-plane. The " $x$-channel" collected the plasma radiation along the width of the current sheet (region D2, $\varnothing \sim 2.5 \mathrm{~cm}$, Figure 1). This channel was used to determine the ions' velocities in the $x$-direction and the 
plasma density, both averaged along the sheet width. Plasma emission from two channels was transmitted via the quartz light guides on the entrance slit of the monochromator MDR-3 (diffraction grating 1200 grooves $/ \mathrm{mm}$, inverse linear dispersion $\mathrm{D}=1.3 \mathrm{~nm} / \mathrm{mm}$ ). The monochromator was completed with the Nanogate 1-UF programmed electro-optical detector. The profiles of the spectral lines were recorded in one pulse of the experimental setup with an integration time of $\approx 0.8$ us.

(a)
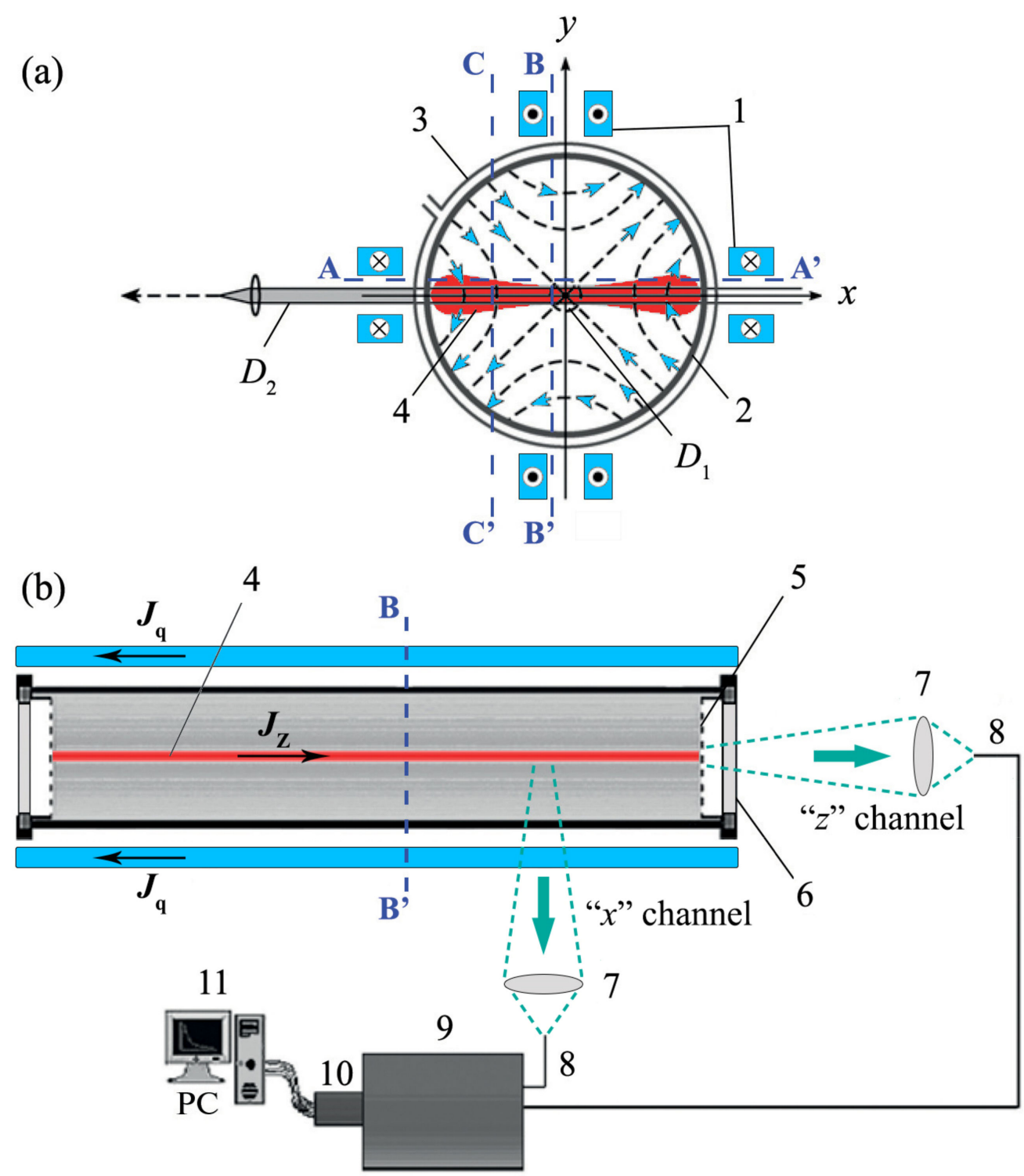

Figure 1. Experimental setup CS-3D (Prokhorov General Physics Institute, Russian Academy of Sciences). (a) Cross section and (b) side view: 1-system of direct conductors with currents for excitation of the 2D magnetic field with the null-line on the $z$ axis: $\vec{B}=\{h y ; h x ; 0\}$; the field lines of the field $\vec{B}$ are shown in the panel (a) by the dashed lines with the arrows; 2 -cylindrical vacuum chamber, its axis is aligned with the null line; 3-system of coils of the $\Theta$ discharge used to create the initial plasma; 4 - current sheet formed after excitation of the plasma current $J_{Z} ; 5$ - grid electrodes; 6-quartz windows; 7-quartz lenses; 8-quartz waveguides; 9-monochromator; 10-Nanogate 1UF electro-optical camera; 11 - computer. $\mathrm{AA}^{\prime}, \mathrm{BB}^{\prime}$, and $\mathrm{CC}^{\prime}$ are the lines of displacement of the magnetic probes. $D_{1}, D_{2}$ - quasi-cylindrical regions from which plasma radiation was detected in the $z$ and $x$ channels, respectively.

In order to investigate the magnetic fields of the current sheets, we recorded the time dependences of three mutually perpendicular components of the magnetic field measured 
by the system of magnetic probes. The probes could be moved in the $x y$-plane either along the width of the current sheet, at a distance of $\approx 1.2 \mathrm{~cm}$ from the $x$-axis (line $\mathrm{AA}^{\prime}$ ), or across the current sheet, at the distances of $-0.8 \mathrm{~cm}$ and $-5.0 \mathrm{~cm}$ from the $y$-axis (lines $\mathrm{BB}^{\prime}$ and $\mathrm{CC}^{\prime}$, respectively). The measured data were then analyzed and processed to obtain the spatial distributions of the magnetic fields, current densities, and the Ampere forces [11,16,21].

\section{Evolution of Plasma Parameters in Current Sheets Formed in the Helium Plasma}

In the context of the presented research, one of the most important characteristics of the current sheet is the energy of plasma flows that are accelerated along the sheet width. The evolution of this energy, along with the evolution of the ion temperature and plasma density, was obtained by analyzing the profiles of the spectral lines: He II $468.6 \mathrm{~nm}$ and He II $320.3 \mathrm{~nm}$. It was found that the broadenings of these lines registered in the $x$-direction $\left(\Delta \lambda_{x}\right)$ were usually several times larger than the broadenings measured in the $z$-direction $\left(\Delta \lambda_{z}\right)[20,33]$. For example, at $t \approx 3.3 \mu \mathrm{s}$, the ratio of the half-widths was $\Delta \lambda_{x} / \Delta \lambda_{z} \approx 4$ for the He II $P_{\beta}$ line, and the same ratio was $\Delta \lambda_{x} / \Delta \lambda_{z} \approx 3$ for the He II $P_{\alpha}$ line at $t \approx 4.3 \mu$ s (Figure 2). The differences between $\Delta \lambda_{x}$ and $\Delta \lambda_{z}$ show that the values of both the ion velocities and plasma density measured in the $x$-channel exceed the same parameters measured in the $z$-channel.

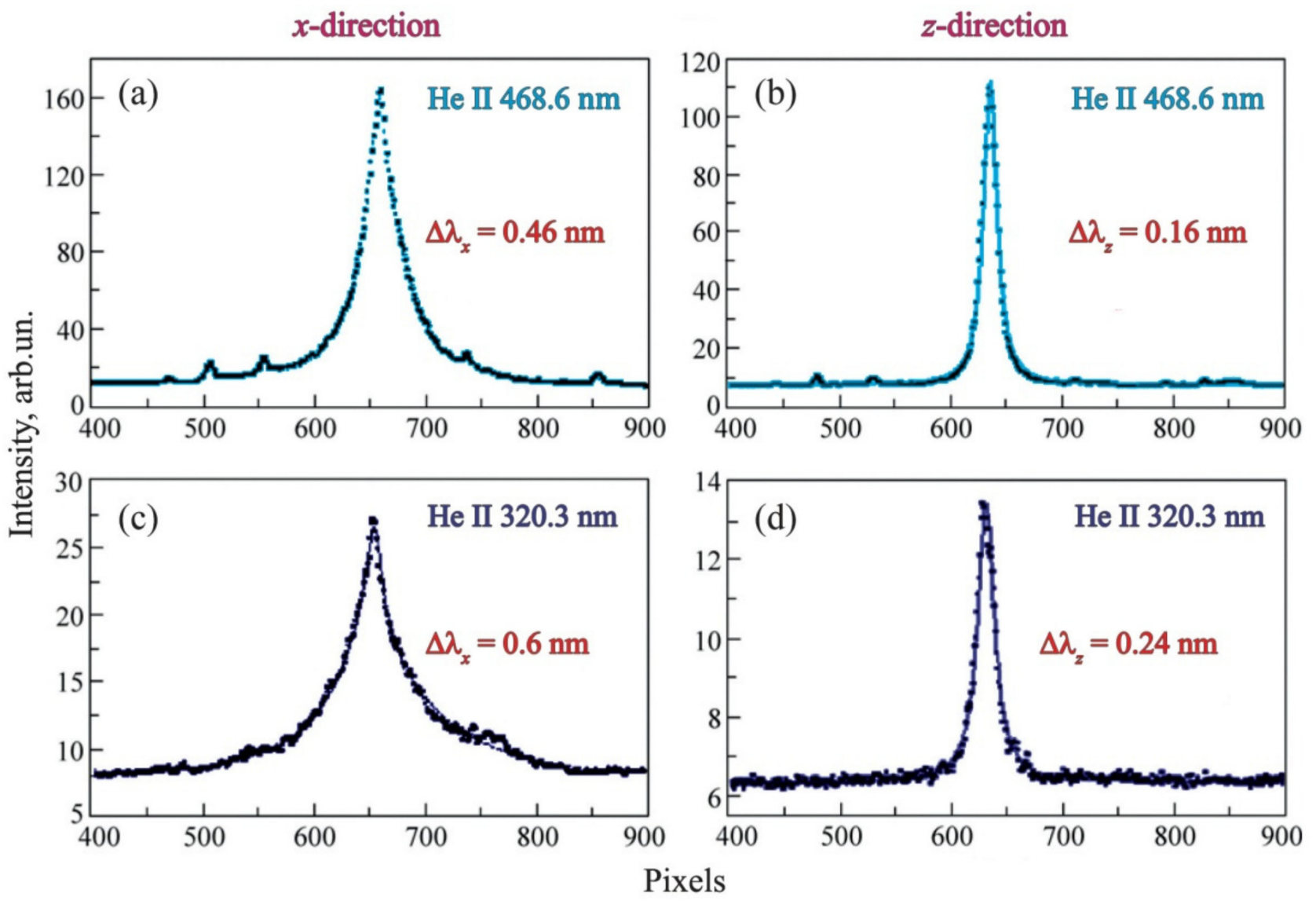

Figure 2. (a,b) - profiles of the He II $468.6 \mathrm{~nm}\left(P_{\alpha}\right)$ spectral line, $(\mathbf{c}, \mathbf{d})$ - profiles of He II $320.3 \mathrm{~nm}\left(P_{\beta}\right)$ spectral line that were emitted by the current sheet plasma at $t=4.3 \pm 0.4 \mu \mathrm{s} ;(\mathbf{a}, \mathbf{c})$-in the $x$-direction, $(\mathbf{b}, \mathbf{d})$-in the $z$-direction [20]. The abscissa axis shows the radiation wavelength $(100$ pixels $=1.25 \mathrm{~nm})$ and the radiation intensity is given on the ordinate axis in arbitrary units. The parameters of the experiment are $h=0.5 \mathrm{kG} / \mathrm{cm}, p=320 \mathrm{mTorr}, J_{Z}^{\max }=45 \mathrm{kA}$.

The quantitative characteristics of the plasma parameters were derived from the experimental data with the use of the Stark constants for the spectral lines He II $P_{\beta}$ [34] and He II $P_{\alpha}$ [35]; in this case, the calculations were carried out by the method of the successive approximations (for details see [33]). Figure 3 shows how plasma parameters 
change with time. The temperature $T_{i}$ of the helium ions and the electron density $N_{e}^{0}$ at the central region of the current sheet were calculated on the base of the data available from the $z$-channel. The values of the ion energy and plasma density $N_{e}^{x}$ calculated on the base of the $x$-channel data were, as a rule, higher than $T_{i}$ and $N_{e}^{0}$.

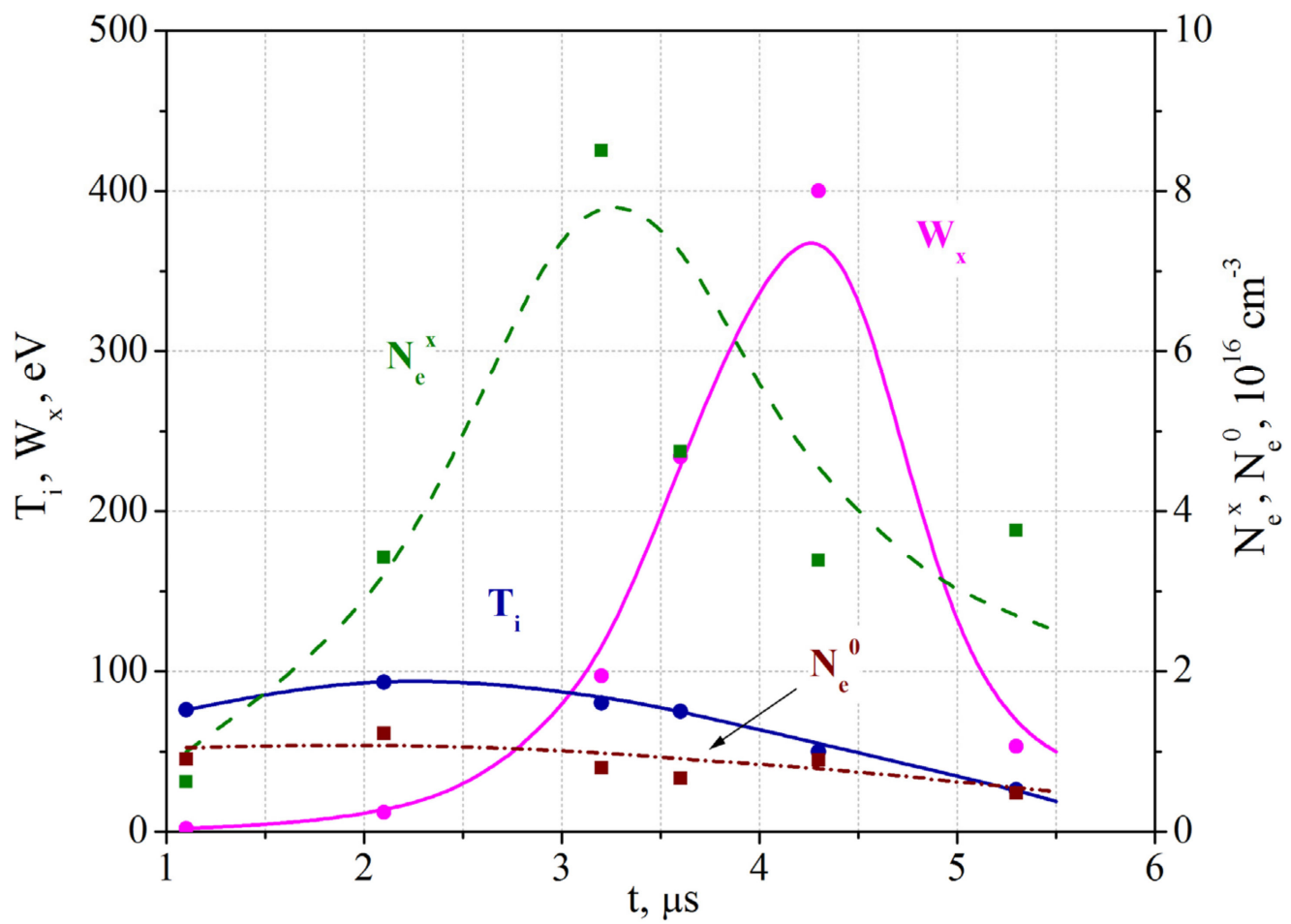

Figure 3. Time dependences of the helium ions' temperature $T_{i}$, the energy of directed motion of helium ions $W_{x}$, and the electron densities $N_{e}^{0}$ and $N_{e}^{x}$ in the central region of the sheet and at its edge, respectively [33]. The experimental conditions are the same as in Figure 2.

The revealed difference between the data from the $x$ - and $z$-channels proves that helium ions receive an excess energy of directed motion along the width of the current sheet (along the $x$-axis) $[20,33]$. We defined the energy of the directed ions' motion $W_{x}$ as the difference between the total ion energy measured by the $x$-channel, and the ion thermal energy $T_{i}$ measured by the $z$-channel at the same time. We also associate the difference between plasma densities $N_{e}^{x}$ and $N_{e}^{0}$ with the plasma motion in the $x$-direction: from the central region of the current sheet to its edges; see below.

One can see from the Figure 3 that, at the early times $(t \approx 1.2 \mu \mathrm{s})$, the electron density was practically uniform along the current sheet, $N_{e}^{0} \approx N_{e}^{x} \approx 10^{16} \mathrm{~cm}^{-3}$, the ion temperature was $T_{i} \approx 90 \mathrm{eV}$, and there were no plasma motions in the $x$-direction: $W_{x} \approx 0$.

The energy $W_{x}$ started increasing after $t \approx 2.2 \mu \mathrm{s}$, and, at $t \approx 3.4 \mu \mathrm{s}, W_{x}$ became equal to the thermal energy: $W_{x} \approx T_{i} \approx 100 \mathrm{eV}$. The corresponding average rate of the energy gain was $\Delta W_{x} / \Delta t \approx 70 \mathrm{eV} / \mu \mathrm{s}$. After this initial stage, the energy of the helium ions started growing much faster: at $t \approx 4.3 \mu \mathrm{s}$, the energy of the plasma directed motions peaked at $W_{x} \approx 400 \mathrm{eV}$, so that the helium ions became superthermal, $W_{x} \gg T_{i} \approx 50 \mathrm{eV}$. At this stage, the rate of the energy gain became $\Delta W_{x} / \Delta t \approx 330 \mathrm{eV} / \mu \mathrm{s}$.

Immediately after the energy of the plasma directed motions attained the maximal value (at $t \approx 4.3 \mu \mathrm{s}$ ), it decreased very quickly; see Figure 3 . In the time interval $t \approx 4.3-5.4 \mu \mathrm{s}$, the rate of the energy decrease was $\left|\Delta W_{x} / \Delta t\right| \approx 320 \mathrm{eV} / \mu \mathrm{s}$. The processes in the current sheet that are responsible for slowing down the accelerated plasma flows are discussed in the next sections of the paper.

The plasma density averaged over the current sheet width, $N_{e}^{x}$, grew significantly at $t>1.5 \mu \mathrm{s}$, and at $t \approx 3.4 \mu \mathrm{s}$ it reached its maximal value, $N_{e}^{x} \approx 8.5 \times 10^{16} \mathrm{~cm}^{-3}$, whereas electron density in the middle of the sheet remained unchanged, $N_{e}^{0} \approx 0.9 \times 10^{16} \mathrm{~cm}^{-3}$. An 
increase in the electron density $N_{e}^{x}$ is an additional indication of plasma motions from the middle of the current sheet towards its edges that resulted in plasma accumulation at the peripheral regions of the sheet. The succeeding decrease in $N_{e}^{x}$ can be related to plasma losses on the walls of the vacuum chamber.

\section{Ampère Forces in Current Sheets and Plasma Acceleration}

Excitation of the plasma current $J_{Z}$ parallel to the null line of the magnetic field (1) brings about formation of a current sheet, i.e., a relatively narrow region compressed in the $y$-direction and elongated in the $x$-direction, where both the electric current and plasma are concentrated. The current sheet formation gives rise to a significant change in the initial magnetic configuration (1): the tangential magnetic field component $B_{x}$ increases significantly near the sheet surface, whereas the normal component $B_{y}$ becomes several times lower than its initial value $B_{y}^{0}$ and holds the same direction as $B_{y}^{0}[21]$.

Typical distributions of the current sheet parameters along the sheet width (along the $x$-direction), shortly after the sheet formation, are shown in the Figure $4 \mathrm{a}$. The presented parameters are the tangential magnetic field component $B_{x}(x)$, the normal magnetic field component $B_{y}(x)$, the linear current $I_{z}(x)=\int j_{z}(x, y) d y$ within the sheet thickness $|y| \leq \Delta y$, and the Ampère force $F_{x}(x)=\int f_{x}(x, y) d y$ within the same region. The linear current $I_{z}(x)$ is maximal in the middle of the sheet (at $x \approx 0$ ), vanishes at the sheet side edges, and in the whole sheet has one, and only one, direction. This direction is specified by the external electric field which had excited the plasma current $J_{Z}$ and given rise to the current sheet formation. The Ampère forces $F_{x}(x)$ are of the opposite directions in the regions $(x>0)$ and $(x<0)$, i.e., the $F_{x}(x)$ forces are directed from the middle of the sheet to both of its side edges (see below). Note that the directions of the Ampère forces $F_{x}(x)$ are marked in Figure 4 by arrows.

Plasma acceleration occurs under the action of a pressure gradient and the Ampère forces:

$$
M_{i} N_{i} \frac{d \vec{v}}{d t}=-\nabla p+\frac{1}{c}[\vec{j} \times \vec{B}] .
$$

Here, $M_{i}, N_{i}$, and $v$ are the mass, concentration, and velocity of plasma ions, $p$ is the plasma pressure, and $\vec{f}=\frac{1}{c}[\vec{j} \times \vec{B}]$ is the density of the Ampère forces. In a current sheet formed in a 2D magnetic field (1), plasma acceleration along the sheet surface (in the $x$-direction) results mainly from the action of the Ampère forces $f_{x}$, since the pressure gradient is usually negligible $[18,29,30]$. The magnitude and direction of the Ampere force $f_{x}$ depend on the current density in the sheet $j_{Z}$ and the strength of the normal magnetic field component $B_{y}$ :

$$
f_{x}(x, y, t)=-\frac{1}{c} j_{z}(x, y, t) \cdot B_{y}(x, y, t)
$$

The Ampère forces $F_{x}(x, t)$ shown on Figure 4 are the integrals of $f_{x}(x, y, t)$ within $|y| \leq \Delta y$ :

$$
F_{x}(x, t)=\int_{-\Delta y}^{\Delta y} f_{x}(x, y, t) d y=-\frac{1}{c} \int_{-\Delta y}^{\Delta y} j_{z}(x, y, t) \cdot B_{y}(x, y, t) d y=-\frac{1}{c} I_{z}(x, t) \cdot B_{y}(x, t)
$$

Here, we take into account that the normal $B_{y}$ component changes insignificantly within the integration region $2 \Delta y[21,28]$. The forces $F_{x}(x)$ can accelerate plasma along the sheet surface, building up the kinetic plasma energy $W_{x}$.

Directed fluxes of helium ions have been actually detected and investigated by spectral methods; see above and References [19,20,33]. Characteristic features of the temporal evolution of the averaged energy of plasma fluxes $W_{x}$ can be followed in Figure 3. During the first $\approx 3.4 \mu$ s after the onset of plasma current $J_{Z}$, the energy $W_{x}$ does not exceed the thermal energy of the helium ions. A sharp increase in the kinetic energy $W_{x}$ starts with a delay which is due mainly to the inertia of the ions [24,28], and, to some extent, to the increase in the Ampère forces with time. As we have shown above, the energy of plasma flows peaks at $t \approx 4.3 \mu$ s, when $W_{x} \approx 400 \mathrm{eV}$, and then goes down. 

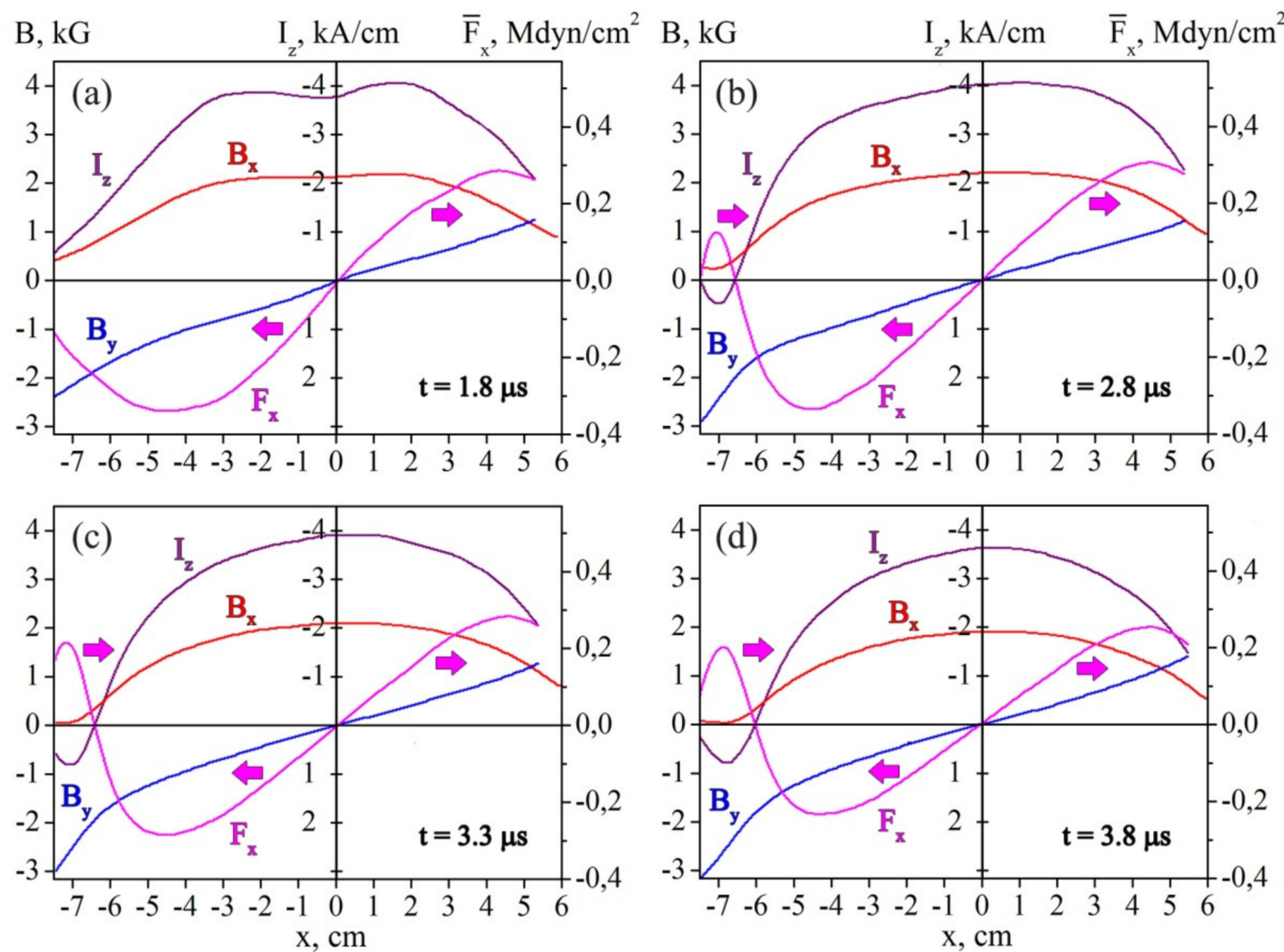

Figure 4. Distributions of the current sheet parameters along the sheet width (along the $x$-direction, at $y=1.2 \mathrm{~cm}$ ) at successive times: (a) $t=1.8 \mu \mathrm{s},(\mathbf{b}) t=2.8 \mu \mathrm{s},(\mathbf{c}) t=3.3 \mu \mathrm{s},(\mathbf{d}) t=3.8 \mu \mathrm{s} . B_{x}(x)$ is the tangential magnetic field component, $B_{y}(x)$ is the normal magnetic field component, $I_{z}(x)=\int j_{z}(x, y) d y$ is the linear current within the sheet thickness $|y| \leq \Delta y=1.2 \mathrm{~cm}$, and $F_{x}(x)=\int f_{x}(x, y) d y$ is the Ampère force within the same region. The directions of the Ampère forces $F_{x}(x)$ are marked by arrows. The experimental conditions are the same as in Figure 2.

In accordance with the structure of the Ampère forces $F_{x}(x)$ shown in Figure $4 \mathrm{a}$, plasma velocities are directed from the central region of the sheet to its side edges, so that the absolute values of the velocities $\left|v_{x}\right|$ should increase near the edges, while, in the central region, the velocities are relatively small and do not exceed the ions' thermal velocities.

\section{Generation of Reverse Currents}

At the later stages of the current sheet evolution, the distributions of the parameters shown in Figure 4 change significantly: see Figure $4 \mathrm{~b}-\mathrm{d}$. A distinctive feature of these distributions is the appearance of reverse currents at the side edges of the current sheet. The reverse currents flow in the direction opposite to the main current in the central region of the sheet. Simultaneously, the current sheet becomes thicker and the current density rapidly decreases at a considerable distance from the null line along the $x$-axis, (compare Figure 5a,b). 


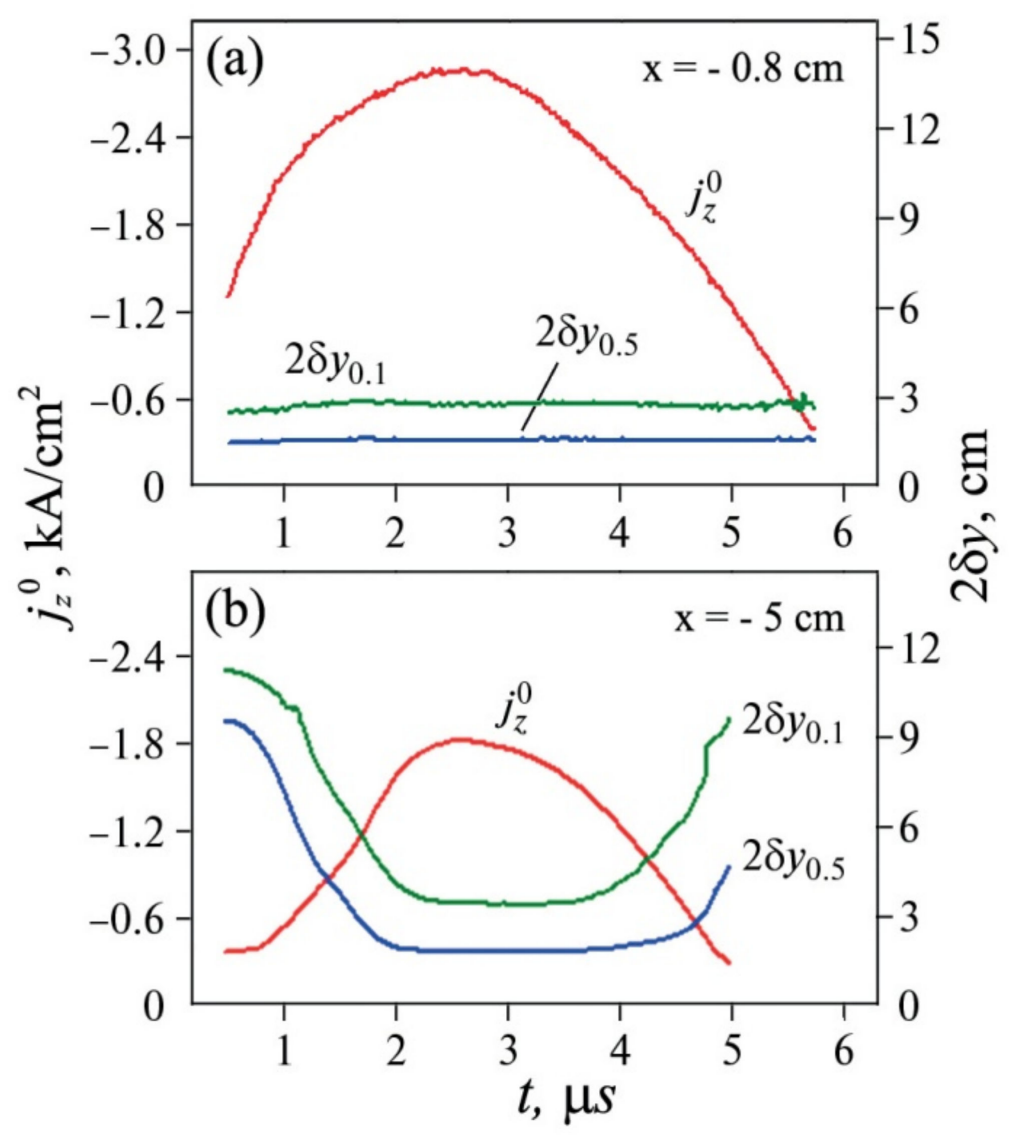

Figure 5. Time dependences of the current density $j_{Z}^{0}$ in the mid-plane of the current sheet $(y=0)$ and transverse sizes of the sheet $2 \delta y_{0.5}$ and $2 \delta y_{0.1}$, determined at the current densities $j_{z}=0.5 j_{Z}^{0}$ and $j_{z}=0.1 j_{Z}^{0}$, respectively, in two cross sections of the sheet: (a) at $x=-0.8 \mathrm{~cm}$ and (b) at $x=-5 \mathrm{~cm}$ [21]. The experimental conditions are the same as in Figure 2.

The possibility of the appearance of reverse currents in current sheets has been predicted theoretically by S.I. Syrovatsky [36], and, subsequently, reverse currents have been revealed experimentally $[11,21]$. The generation of reverse currents is closely connected with the motion of plasma flows in the magnetic field of the current sheet, as we have demonstrated for current sheets formed in plasmas with heavy ions-Ar and $\mathrm{Kr}$ [24,37]. In this paper we present results related to phenomena that occur in current sheets formed in plasma containing the He ions. We consider the excitation of reverse currents, as well as the effect of reverse currents on the dynamics of plasma flows.

It is known that plasma motion in a magnetic field gives rise to the excitation of inductive electric fields. This effect should also come into play when plasma flows move along the width of the current sheet across the transverse magnetic field $\left|B_{y}\right|$. This motion should result in the generation of the inductive electric fields $E_{z}{ }^{\prime}$ :

$$
E_{z}^{\prime}(x)=\frac{1}{c} v_{x}(x) \cdot B_{y}(x),
$$

where $v_{x}(x)$ is the velocity of the plasma flow and $B_{y}(x)$ is the transverse magnetic field.

Within the whole current sheet, the electric field $E_{z}{ }^{\prime}$ is directed oppositely to the initial field $E_{z}^{0}$, which has caused the plasma current $J_{Z}$ and the current sheet formation. As follows from the relation (5), the inductive fields $E_{z}^{\prime}(x)$ should be substantially inhomogeneous within the width of the sheet. This occurs because both the velocities of the plasma flows and the strength of the normal component of the magnetic field are inhomogeneous [24]. The maximal values of the field $E_{z}^{\prime}(x)$ should be reached at the side edges, where both absolute values $\left|v_{x}(x)\right|$ and $\left|B_{y}(x)\right|$ are sufficiently large, so that the inductive fields here can exceed the absolute value of the initial electric field $E_{z}^{0}$. As a result, 
currents $j_{Z}{ }^{\prime}$ of the opposite direction with respect to the main current $j_{Z}$ are excited in the sheet, and this phenomenon is most clearly manifested at the side edges of the sheet.

Moreover, a faster decrease in the current density $j_{Z}^{0}$ and increase in the current sheet thickness, $28 y$, at the considerable distance from the null line along the $x$-axis, Figure $5 \mathrm{~b}$, are also due to the appearance of reverse currents, but of a smaller value than at the sheet edges; see also [24].

Excitation of currents of the opposite direction at the side edges of the current sheet generates the Ampère forces $F_{x}{ }^{\prime}$ in these regions, which are directed oppositely to the forces in the sheet central region, i.e., from the side edges to the middle of the sheet; Figure $4 \mathrm{~b}-\mathrm{d}$. The absolute values of the forces $F_{x}{ }^{\prime}$ are comparable with the forces $F_{x}$ in the middle of the sheet, despite that the reverse currents are relatively small. This is due to the significant strength of the normal $B_{y}$ component at the sheet side edges. The appearance of the forces $F_{x}{ }^{\prime}$ should result in changing plasma dynamics in the current sheet, and can produce deceleration of previously accelerated plasma flows.

\section{Work of the Ampère Forces and Slow down of Plasma Flows}

The kinetic energy of plasma flows can change (either increase or decrease) due to the work of the Ampère forces at the distances where these forces act. Below, we estimate the work of the Ampère forces $F_{x}(x)$ at the half-width of the current sheet $\left(0 \leq|x| \leq\left|R_{\mathcal{C}}\right|\right)$ at successive times:

$$
A(t)=\int_{-R_{c}}^{0} F_{x}(x, t) d x .
$$

Here, $R_{c}$ is the radius of the vacuum chamber, i.e., the largest possible half-width of the sheet. The values of the work $A(t)$ are calculated separately for two different regions. The first is the central region $\left(0 \leq|x| \leq\left|x^{R}(t)\right|\right)$, where the main (direct) currents are concentrated, and we denote the work of Ampère forces at this region as $A_{D}$; Figure 6a. At the second region $\left(\left|x^{R}(t)\right| \leq|x| \leq\left|R_{c}\right|\right)$, the reverse currents are concentrated, and this work is denoted as $A_{R}$; Figure 6a. Here, $x^{R}(t)$ is the coordinate where both the linear current $I_{z}(x, t)$ and the Ampère force $F_{x}(x, t)$ change directions; see Figure 4 . The total work of the Ampere forces $A_{T}$ within the whole half-width of the current sheet $\left(0 \leq|x| \leq\left|R_{c}\right|\right)$ is also shown in Figure 6a; it is evident that $A_{T}=A_{D}+A_{R}$.

At the first stage of the current sheet evolution $(t<4.0 \mu \mathrm{s})$, the work of the Ampère forces is positive, $A_{T} \approx A_{D}$, Figure $6 \mathrm{a}$, and plasma can be effectively accelerated along the sheet surface. Then, the situation changes dramatically, since the absolute value of the work $\left|A_{R}\right|$ sharply increases due to the excitation of reverse currents. Simultaneously, the $A_{D}$ value slightly decreases; so that the total work $A_{T}$ goes down and, moreover, it even becomes negative after $t \approx 5.0 \mu \mathrm{s}$. Based on these results, we can conclude that, at the later stages of the current sheet evolution, the plasma flows should slow down.

The data presented in Figure 6a allow us to give an interpretation for experimental results related to the evolution of the kinetic energy of plasma flows; see Figure $6 \mathrm{~b}$. A comparison of the work of the multidirectional Ampère forces $A_{D}$ and $A_{R}$, on the one hand, with changes in the energy of the accelerated plasma flows $W_{x}$, on the other hand, demonstrate a satisfactory time correlation; see Figure 6. Plasma is effectively accelerated in the first stage of the current sheet evolution, when the work of the Ampère forces $A_{D}>>A_{R}$, and the total work is positive, $A_{T}>>0$. At this stage, plasma flows become super-thermal. Later on, the excitation of reverse currents and the appearance of significant Ampere forces $F_{x}{ }^{\prime}$ caused by the reverse currents result in braking previously accelerated plasma flows and a rapid decrease in the energy $W_{x}$.

The appearance of braking forces makes it possible to explain the nature of the temporal changes in the energy of plasma flows, when the energy achieves the superthermal value and then reduces quite quickly $[20,23,24,33]$. 

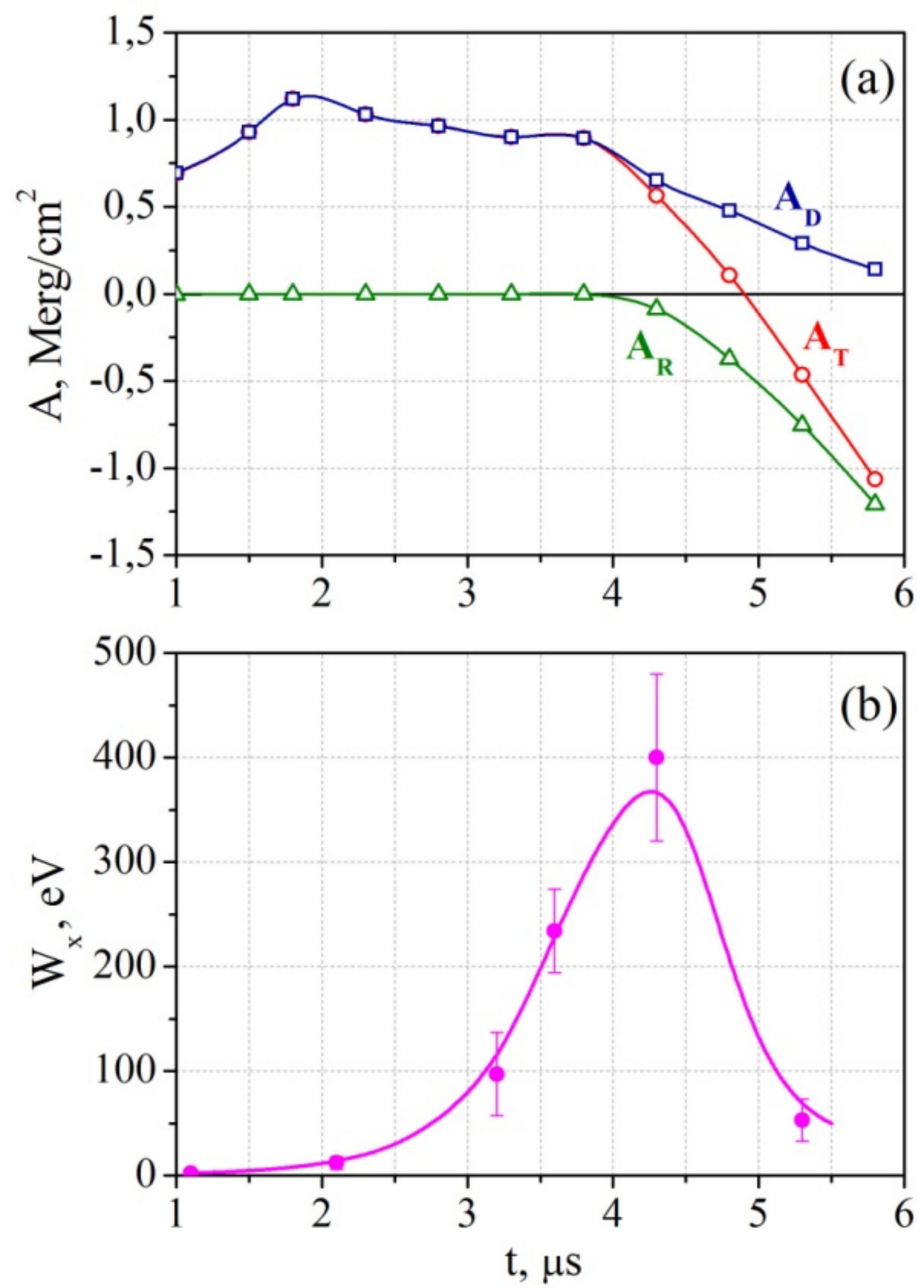

Figure 6. Time dependences of the work of the Ampère forces $A(t)=\int F_{x}(x, t) d x(\mathbf{a})$ and of the energy of accelerated plasma flows $W_{x}(t)(\mathbf{b}) . A_{D}$ is the work within the region $\left(x^{R}(t) \leq x \leq 0\right)$ where the main (direct) currents are concentrated; $A_{R}$ is the work within the region $\left(-R_{c} \leq x \leq x^{R}(t)\right)$ where the reverse currents are concentrated; $A_{T}=A_{D}+A_{R}$ is the work within the half-width of the sheet $\left(-R_{c} \leq x \leq 0\right)$. At $x^{R}(t)$, the directions of both the linear current $I_{z}(x, t)$ and the Ampere force $F_{x}(x, t)$ are changed. The experimental conditions are the same as in Figure 2.

It can be assumed that the generation of reverse currents and, as a result, the appearance of braking forces, can also affect the characteristics of high-speed plasma flows that extend from the tail region of the magnetosphere towards the Earth. Indeed, as shown by direct measurements carried out with the help of various satellite missions, the deceleration of plasma flows directed to the Earth has been recorded at distances from the Earth about (10-20) $R_{E}$ [38-41] (here, $R_{E}$ is the radius of the Earth). We believe that the excitation of the reverse currents and the appearance of additional forces directed towards high-speed plasma flows can be a significant factor leading to slowing down plasma flows in the Earth's magnetosphere.

\section{Conclusions}

In this paper, we present experimental results on the dynamics of plasma with the helium ions in the current sheets formed in two-dimensional magnetic fields with the null-line of the X-type. The research was carried out using the CS-3D experimental setup at the Prokhorov General Physics Institute of the Russian Academy of Sciences. The parameters of the plasma in the current sheets were investigated by the spectroscopic methods using two various spectral lines of the helium ion that were registered along 
two mutual perpendicular directions. The structure and evolution of the magnetic fields, electric currents, and the Ampère forces in the current sheets were studied using a system of magnetic probes.

We demonstrate an appearance of plasma flows accelerated along the width (the larger transverse size) of the current sheet. The energy of the flows increases rapidly and peaks at $400 \mathrm{eV}$, whereas the temperature of the helium ions is about $50 \mathrm{eV}$. Immediately upon reaching its maximum, the energy of the plasma flows decreases very quickly.

We analyze the structure of the Ampère forces that can accelerate plasma along the current sheet width. At the early stage of the current sheet evolution, the Ampère forces are directed from the middle of the sheet to both of its side edges, and build up the kinetic energy of directed flows of the helium ions.

The plasma flows that are moving in the magnetic field of the current sheet excite the inductive electric fields and the electric currents of the opposite directions relative to the main current in the sheet. The reverse currents reveal themselves primarily at the side edges of the current sheet, and bring about the Ampère forces that are directed oppositely to the forces in the sheet central region. These forces modify plasma dynamics in the current sheet, initiating deceleration of previously accelerated plasma flows. Comparison of the work of the multidirectional Ampère forces in the current sheet with the evolution of the energy of the accelerated plasma flows demonstrates a satisfactory time correlation.

The appearance of braking forces makes it possible to explain the nature of the temporal changes in the energy of plasma flows, when the energy has achieved the superthermal value and then reduces quite quickly.

It should be emphasized that phenomena related to the excitation of reverse currents in the current sheets represent a manifestation of the general Lenz's rule. According to this rule, the induction current always results in decreasing the impact of the cause that has excited this current.

It seems reasonable that the excitation of the reverse currents and the appearance of the Ampère forces directed towards high-speed plasma flows can be a significant factor leading to braking plasma flows in the Earth's magnetosphere.

Author Contributions: Conceptualization: A.G.F.; methodology: N.P.K., A.G.F.; software: S.N.S.; validation: A.G.F., S.A.S.; formal analysis: S.A.S.; investigation: N.P.K.; S.N.S.; data curation: S.N.S., S.A.S.; writing-original draft preparation: N.P.K., A.G.F.; writing-review and editing: A.G.F.; visualization: S.A.S. All authors have read and agreed to the published version of the manuscript.

Funding: This research received no external funding.

Conflicts of Interest: The authors declare no conflict of interest.

\section{References}

1. Syrovatskii, S.I. Pinch sheets and reconnection in astrophysics. Annu. Rev. Astron. Astrophys. 1981, 19, 163-229. [CrossRef]

2. Biscamp, D. Magnetic Reconnection in Plasmas, 2nd ed.; Cambridge University Press: Cambridge, UK, 2005; 404p.

3. Priest, E.R.; Forbes, T. Magnetic Reconnection. MHD Theory and Applications, 1st ed.; Cambridge University Press: Cambridge, UK, 2000; 600p.

4. Zelenyi, L.M.; Artemyev, A.V.; Malova, K.V.; Petrukovich, A.A.; Nakamura, R. Metastability of current sheets. Phys.-Usp. 2010, 53, 933-941. [CrossRef]

5. Kadomtsev, B.B. Reconnection of Magnetic Force Lines. Usp. Fiz. Nauk 1987, 151, 3-29. [CrossRef]

6. Zweibel, E.G.; Yamada, M. Magnetic reconnection in Astrophysical and Laboratory Plasmas. Annu. Rev. Astron. Astrophys. 2009, 47, 291-332. [CrossRef]

7. Frank, A.G. Dynamics of current sheets underlying flare-type events in magnetized plasmas. Phys.-Usp. 2010, 180, 941-947. [CrossRef]

8. Yamada, M.; Kurlsrud, R.; Ji, H. Magnetic Reconnection. Rev. Mod. Phys. 2010, 82, 603-664. [CrossRef]

9. Artemyev, A.V.; Petrukovich, A.A.; Frank, A.G.; Vasko, I.Y.; Nakamura, R.; Zelenyi, L.M. Intense current sheets in the magnetotail: Peculiarities of electron physics. J. Geophys. Res. 2013, 118, 2789-2799. [CrossRef]

10. Frank, A.G.; Artemyev, A.V.; Zelenyi, L.M. Current sheets in the Earth's magnetosphere and in laboratory experiments: The magnetic field structure and the Hall effect. J. Exp. Theor. Phys. 2016, 123, 699-715. [CrossRef] 
11. Frank, A.G.; Kyrie, N.P.; Satunin, S.N. Plasma dynamics in laboratory-produced current sheets. Phys. Plasmas 2011, 18, 111209. [CrossRef]

12. Yushkov, E.V.; Frank, A.G.; Artemyev, A.V.; Petrukovich, A.A.; Vasko, I.Y. Formation of a Quasi-One-Dimensional Current Sheet in the Laboratory Experiment and in the Earth's Magnetotail. Plasma Phys. Rep. 2015, 41, 71-87. [CrossRef]

13. Zelenyi, L.M.; Frank, A.G.; Artemyev, A.V.; Petrukovich, A.A.; Nakamura, R. Formation of Sub-Ion Scale Filamentary Force-Free Structures in Vicinity of Reconnection Region. Plasma Phys. Controll. Fusion 2016, 58, 054002. [CrossRef]

14. Frank, A.G.; Ostrovskaya, G.V.; Yushkov, E.V.; Artemyev, A.V.; Satunin, S.N. Structure of Current and Plasma in Current Sheets Depending on the Conditions of Sheet Formation. Cosm. Res. 2017, 55, 46-56. [CrossRef]

15. Yushkov, E.V.; Frank, A.G.; Artemyev, A.V.; Petrukovich, A.A.; Nakamura, R. Hall Effect in Laboratory and Space Current Sheets. Plasma Phys. Rep. 2018, 44, 1126-1134. [CrossRef]

16. Frank, A.G.; Satunin, S.N. Structure of longitudinal and transverse currents in current sheets. Plasma Phys. Rep. 2018, 44, 190-202. [CrossRef]

17. Frank, A.G.; Satunin, S.N. Some Features of Current Sheets Formed in Plasmas with Ions of Different Mass. Bull. Lebedev Phys. Inst. 2020, 47, 54-58. [CrossRef]

18. Voronov, G.S.; Kyrie, N.P.; Markov, V.S.; Ostrovskaya, G.V.; Frank, A.G. Spectroscopic Measurements of the Electron and Ion Temperatures and Effective Ion Charge in Current Sheets Formed in Two- and Three-Dimensional Magnetic Configurations. Plasma Phys. Rep. 2008, 34, 999-1015. [CrossRef]

19. Kyrie, N.P.; Markov, V.S.; Frank, A.G. Suprathermal Plasma Flows in Current Sheets Formed in Two- and Three-Dimensional Magnetic Configurations. Plasma Phys. Rep. 2010, 36, 357-364. [CrossRef]

20. Kyrie, N.P.; Markov, V.S.; Frank, A.G. Generation of Superthermal Plasma Flows in Current Sheets. JETP Lett. 2012, 95, 14-19. [CrossRef]

21. Frank, A.G.; Satunin, S.N. Dynamics of the structure of electric currents and electrodynamic forces in current sheets. Plasma Phys. Rep. 2011, 37, 829-847. [CrossRef]

22. Frank, A.G.; Kyrie, N.P. Experimental Studies of the Magnetic Structure and Plasma Dynamics in Current Sheets (Review). Plasma Phys. Rep. 2017, 43, 696-710. [CrossRef]

23. Kyrie, N.P.; Savinov, S.A. Spectroscopic Investigation of Tangential Acceleration and Heating of Plasma of Current Sheets Formed in the Krypton Discharge. Plasma Phys. Rep. 2021, 47, 611-622. [CrossRef]

24. Frank, A.G.; Satunin, S.N. On the Generation of Opposite Currents in Current Sheets. JETP Lett. 2020, 112, 623-629. [CrossRef]

25. Frank, A.G. Magnetic Reconnection and Currents Sheet Formation in 3D Magnetic Configurations. Plasma Phys. Contr. Fusion 1999, 41, A687-A697. [CrossRef]

26. Bogdanov, S.Y.; Kyrie, N.P.; Markov, V.S.; Frank, A.G. Current sheets in magnetic configurations with singular X-lines. JETP Lett. 2000, 71, 53-57. [CrossRef]

27. Bogdanov, S.; Yu, G.; Bugrov, S.G.; Gritsyna, V.P.; Zverev, O.V.; Karpov, G.V.; Marko, V.S.; Repin, D.V.; Frank, A.G. Magnetic Structure of Current Sheets in Magnetic Fields with a Singular X line. Plasma Phys. Rep. 2007, 33, 435-443. [CrossRef]

28. Frank, A.G.; Bugrov, S.G.; Markov, V.S. Hall currents in a current sheet: Structure and dynamics. Phys. Plasmas 2008, 15, 092102. [CrossRef]

29. Bogdanov, S.Y.; Markov, V.S.; Frank, A.G.; Dreiden, G.V.; Komissarova, I.I.; Ostrovskaya, G.V.; Shedova, E.N. Study of Current Sheets in Three-Dimensional Magnetic Fields with an X-line by Holographic Interferometry. Plasma Phys. Rep. 2002, 28, 549-558. [CrossRef]

30. Frank, A.G.; Bogdanov, Y.S.; Markov, V.S.; Dreiden, G.V.; Ostrovskaya, G.V. Experimental study of plasma compression into the sheet in three-dimensional magnetic fields with singular X lines. Phys. Plasmas 2005, 12, 052316. [CrossRef]

31. Griem, H.R. Plasma Spectroscopy, 1st ed.; McGraw-Hill: New York, NY, USA, 1964; p. 580.

32. Griem, H.R. Spectral Line Broadening by Plasmas, 1st ed.; Academic Press: New York, NY, USA, $1974 ;$ p. 424.

33. Kyrie, N.P.; Frank, A.G. Plasma Acceleration in Current Sheets Formed in Helium in Two- and Three-Dimensional Magnetic Configurations. Plasma Phys. Rep. 2012, 38, 960-971. [CrossRef]

34. Ackermann, U.; Finken, K.H.; Musielok, J. Line Profiles of the P $\alpha$ and P $\beta$ Transitions in He II and broadening of C IV Lines at High Electron Densities. Phys. Rev. A 1985, 31, 2597-2609. [CrossRef]

35. Pittman, T.L.; Fleurier, C. Plasma Shifts of the He II H $\alpha$ and $\mathrm{P} \alpha$ Lines. Phys. Rev. A 1986, 33, 1291-1296. [CrossRef] [PubMed]

36. Syrovatskii, S.I. Formation of Current Sheets in a Plasma with a Frozen-In Strong Magnetic Field. Sov. Phys. JETP 1971, 33, 933-940.

37. Frank, A.G.; Satunin, S.N. Reverse currents in current sheets and braking of plasma flows. Plasma Phys. Rep. $2022,48$.

38. Baumjohann, W.; Paschmann, G.; Luehr, H. Characteristics of high-speed ion flows in the plasma sheet. J. Geophys. Res. 1990, 95, 3801-3809. [CrossRef]

39. Angelopoulos, V.; Baumjohann, W.; Kennel, C.F.; Coroniti, F.V.; Kivelson, M.G.; Pellat, R.; Walker, R.J.; Luehr, H.; Paschmann, G. Bursty Bulk Flows in the Inner Central Plasma Sheet. J. Geophys. Res. 1992, 97, 4027-4029. [CrossRef]

40. Shiokawa, K.; Baumjohann, W.; Haerendel, G. Braking of highspeed flows in the near-Earth tail. Geophys. Res. Lett. 1997, 24, 1179-1182. [CrossRef]

41. Birn, J.; Hesse, M.; Haerendel, G.; Baumjohann, W.; Shiokawa, K. Flow braking and the substorm current wedge. J. Geophys. Res. 1999, 104, 19895-19904. [CrossRef] 